\title{
ON STIELTJES AND VAN VLECK POLYNOMIALS
}

\author{
NEYAMAT ZAHEER
}

\begin{abstract}
Stieltjes and Van Vleck polynomials arise in the study of the polynomial solutions of the generalized Lamé differential equation. The problem of determining the location of the zeros of such polynomials has been studied under quite general conditions by Marden. He has obtained (see Trans. Amer. Math. Soc. 33 (1931), 934-944) varied generalizations of certain results proved earlier by Stieltjes, Van Vleck, Bôcher, Klein, and Pólya. Our object in this paper is to study certain aspects of the corresponding problem in relation to yet another form of the generalized Lamé differential equation. Furthermore, applications of our theorems to the standard form of the generalized Lamé differential equation immediately furnish the corresponding results due to Stieltjes, Van Vleck, and Marden (cf. the paper cited above).
\end{abstract}

1. Introduction. Heine [2] has shown that there exist at most $C(n+p-2, p$ - 2) polynomials $V(z)$ with $\operatorname{deg} V \leqslant p-2$ such that, for $\Phi(z)=V(z)$, the generalized Lamé differential equation

$$
w^{\prime \prime}+\left[\sum_{j=1}^{p} \frac{\alpha_{j}}{z-a_{j}}\right] \cdot w^{\prime}+\left[\Phi(z) / \prod_{j=1}^{p}\left(z-a_{j}\right)\right] \cdot w=0
$$

has a polynomial solution $S(z)$ of degree $n$. Such $S(z)$ and $V(z)$ are called [5, pp. 36-37] Stieltjes and Van Vleck polynomials, respectively. We observe that the differential equation

$$
\begin{gathered}
w^{\prime \prime}+\left[\sum_{j=1}^{p} \alpha_{j}\left\{\prod_{t=1}^{n_{j}-1}\left(z-b_{j t}\right) / \prod_{s=1}^{n_{j}}\left(z-a_{j s}\right)\right\}\right] \cdot w^{\prime} \\
+\frac{\Phi(z)}{\prod_{j=1}^{p} \prod_{s=1}^{n_{j}}\left(z-a_{j s}\right)} \cdot w=0,
\end{gathered}
$$

where $\Phi(z)$ is a polynomial of degree at most $\left(n_{1}+\cdots+n_{p}-2\right)$, can always be written in the form (1.1). (Note that (1.2) is indeed of the form (1.1) if $n_{j}=1$ for all $j$.)

Various mathematicians (see Marden [6, pp. 935-936]) have, via different methods, studied the zeros of the polynomials $S(z)$ and $V(z)$ in relation to the differential equation (1.1) by imposing suitable conditions on the singularities

Received by the editors December 8, 1975 and, in revised form, March 27, 1976.

AMS (MOS) subject classifications (1970). Primary 30A08; Secondary 33A70.

Key words and phrases. Generalized Lamé differential equations, Stieltjes polynomials, Van Vleck polynomials. 
$a_{j}$, but only for positive real values of the constants $\alpha_{j}$. For the first time, Marden [6] gave the treatment of (1.1) subject to condition $\left|\arg \alpha_{j}\right| \leqslant \gamma<\pi / 2$ and obtained varied generalizations of the results (cf. Marden [6, Theorems 1(a)-2(a)]) established earlier by Stieltjes [8], Van Vleck [9], Bôcher [1], Klein [3], and Pólya [7]. Our object in this paper is to study the zeros of the polynomials $S(z)$ and $V(z)$ in relation to the differential equation (1.2). The results thus obtained are valid for both (1.1) and (1.2), whereas the corresponding known results [6, Theorems $1(\mathrm{a}), 1(\mathrm{~b}), 6(\mathrm{~b})]$, which apply only to (1.1), become corollaries to our theorems. However, the present treatment follows, in some aspects, the methods introduced by Marden [6, pp. 934-937].

2. Main theorems. Throughout this section, we shall freely use the following abbreviations:

$$
f_{j}(z)=\prod_{t=1}^{n_{j}-1}\left(z-b_{j t}\right), \quad g_{j}(z)=\prod_{s=1}^{n_{j}}\left(z-a_{j s}\right), \quad h_{j}(z)=\frac{f_{j}(z)}{g_{j}(z)}
$$

for every $1 \leqslant j \leqslant p$ (with the convention that $f_{j}(z) \equiv 1$ whenever $n_{j}=1$ ), and

$$
F(z)=\sum_{j=1}^{p} \alpha_{j} \cdot h_{j}(z)
$$

We intend to prove

THEOREM (2.1). If $\left|\arg \alpha_{j}\right| \leqslant \gamma<\pi / 2$ and if all the points $a_{j s}, b_{j t}$ (occurring in (1.2)) lie on the line segment joining the points $c_{1}$ and $c_{2}$, then the zeros of each Stieltjes polynomial $S(z)$, associated with the differential equation (1.2), lie in the region $K$ given by

$$
K=\left\{z|| z-c_{1}|+| z-c_{2}|\leqslant| c_{1}-c_{2} \mid \cdot \sec \mu\right\}
$$

where

$$
\mu=\{(q-1) \pi+\gamma\} /(2 q-1), \quad q=\max \left\{n_{1}, n_{2}, \ldots, n_{p}\right\}
$$

Proof. If $S(z)=\left(z-z_{1}\right)\left(z-z_{2}\right) \cdots\left(z-z_{n}\right)$ is a Stieltjes polynomial corresponding to a Van Vleck polynomial $V(z)$, associated with (1.2), then we know [10, Lemma (2.1)] that every zero $z_{k}$ of $S(z)$ is either a point $a_{j s}$ or satisfies the equation

$$
\frac{1}{2} F\left(z_{k}\right)+\sum_{j \neq k, j=1}^{n} \frac{1}{z_{k}-z_{j}}=0 \quad(k=1,2, \ldots, n),
$$

where $F(z)$ is as defined by (2.2).

Suppose, on the contrary, that one or more zeros $z_{1}, \ldots, z_{m}$ (say) of $S(z)$ lie outside the region $K$. Let us consider the family $\mathscr{F}$ of all confocal ellipses having foci at the points $c_{1}$ and $c_{2}$. Then there passes (through each point in 
the complex plane) a unique member of $\mathscr{F}$ and no two distinct members of $\mathscr{F}$ intersect one another. If $E_{1}, E_{2} \in \mathscr{F}$ (eccentricities $e_{1}, e_{2}$ respectively), then either $E_{1}=E_{2}$ (with $e_{1}=e_{2}$ ), or else one of them (say, $E_{1}$ ) falls inside the other (say, $E_{2}$ ) and in that case $e_{1}>e_{2}$. Consequently, out of the zeros $z_{1}, \ldots, z_{m}$ there is at least one (say, $z_{1}$ ) such that a member $E_{e}$ of $\mathscr{F}$ (with eccentricity $e$ ) passes through $z_{1}$ and such that all the zeros $z_{1}, \ldots, z_{n}$ lie on or inside $E_{e}$. Notice that either $K$ is the line segment with endpoints $c_{1}$ and $c_{2}$ (in case $\mu=0$ ) or else that the boundary of $K$ is a member of $\mathscr{F}$ having eccentricity $\cos \mu$ (in case $\mu>0$ ). Since $K \varsubsetneqq E_{e}$, in either case, we conclude that

$$
e<\cos \mu \text {. }
$$

Also, since $0 \leqslant \gamma<\pi / 2$ and $n_{j} \leqslant q$, we see that $[(n-1) \pi+\gamma] /(2 n-1)$ is an increasing function of $n$ and that

$$
0 \leqslant \frac{\left(n_{j}-1\right) \pi+\gamma}{\left(2 n_{j}-1\right)}=\mu_{j} \quad(\text { say }) \leqslant \mu<\frac{\pi}{2}, \quad 1 \leqslant j \leqslant p .
$$

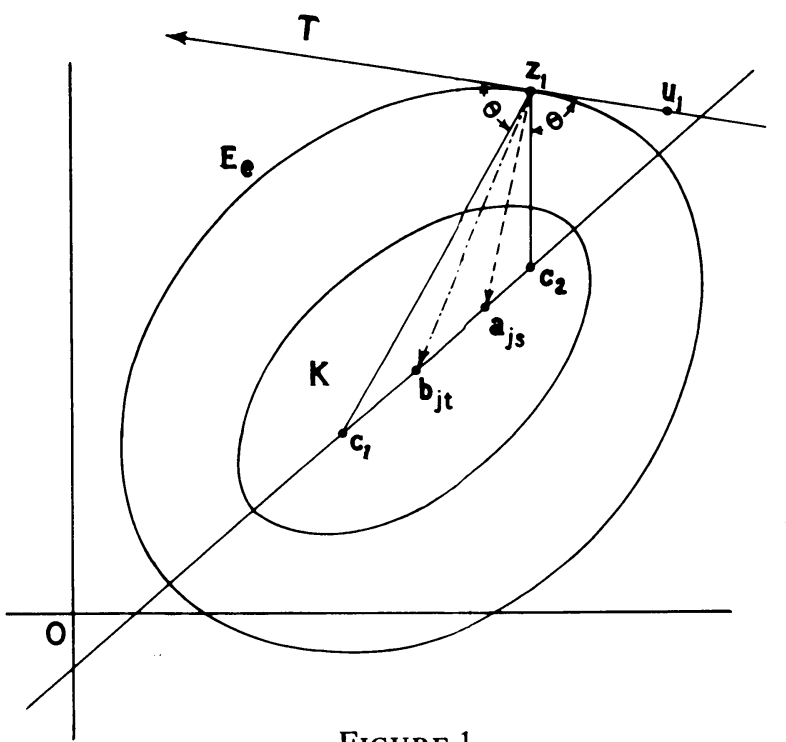

Figure 1

Let us take a fixed point $u_{1}$ on the tangent $T$ at $z_{1}$ to the ellipse $E_{e}$ (see Figure 1) and draw straight lines joining $z_{1}$ to the foci $c_{1}, c_{2}$. These lines obviously make equal angles $\theta$ (say) with the tangent $T$. By elementary calculus, we can easily verify that the minimum value $\theta_{0}$ of $\theta$ (with respect to all positions of the point $z_{1}$ on the ellipse $\left.E_{e}\right)$ is given by $\theta_{0}=\cos ^{-1} e$, which is attained when $z_{1}$ is at an end of the minor-axis. Now inequalities (2.4) and (2.5) imply that

$$
\theta>\mu_{j} \quad \forall j=1,2, \ldots, p
$$


Returning to the proof of our main theorem, we notice that a zero $z_{k}$ of $S(z)$ does lie in $K$ if $z_{k}$ is one of the points $a_{j s}$. If a zero $z_{k}$ is none of the points $a_{j s}$, then $z_{k}$ satisfies (2.3). In particular (for $k=1$ ), we have

$$
\frac{1}{2} \cdot F\left(z_{1}\right)+\sum_{j=2}^{n} \frac{1}{z_{1}-z_{j}}=0
$$

Therefore,

$$
\frac{1}{2} \cdot F\left(z_{1}\right) \cdot\left(z_{1}-u_{1}\right)+\sum_{j=2}^{n} \frac{z_{1}-u_{1}}{z_{1}-z_{j}}=0
$$

i.e.

$$
\sum_{j=1}^{p}\left[\frac{\alpha_{j}}{2} \cdot \prod_{t=1}^{n_{j}-1}\left(\frac{z_{1}-b_{j t}}{z_{1}-u_{1}}\right) \cdot \prod_{s=1}^{n_{j}}\left(\frac{z_{1}-u_{1}}{z_{1}-a_{j s}}\right)\right]+\sum_{j=2}^{n}\left(\frac{z_{1}-u_{1}}{z_{1}-z_{j}}\right)=0
$$

Since all the zeros $z_{k}$ lie on or inside the ellipse $E_{e}$, we have

$$
0<\arg \left(\frac{z_{1}-u_{1}}{z_{1}-z_{j}}\right)<\pi \quad \forall j=2,3, \ldots, n .
$$

Also, due to inequality (2.6) and the hypotheses on $a_{j s}$ and $b_{j t}$, we have (for $1 \leqslant j \leqslant p$ )

$$
\begin{gathered}
-\left(\pi-\mu_{j}\right)<-(\pi-\theta) \leqslant \arg \left(\frac{z_{1}-b_{j t}}{z_{1}-u_{1}}\right) \leqslant-\theta<-\mu_{j}, \\
\mu_{j}<\theta \leqslant \arg \left(\frac{z_{1}-u_{1}}{z_{1}-a_{j s}}\right) \leqslant \pi-\theta<\pi-\mu_{j}
\end{gathered}
$$

for every $t=1,2, \ldots, n_{j}-1$ and $s=1,2, \ldots, n_{j}$. Consequently,

$$
\begin{array}{r}
-\left(n_{j}-1\right) \cdot\left(\pi-\mu_{j}\right) \leqslant \arg \left[\prod_{t=1}^{n_{j}-1}\left(\frac{z_{1}-b_{j t}}{z_{1}-u_{1}}\right)\right] \leqslant-\left(n_{j}-1\right) \mu_{j}, \\
n_{j} \mu_{j}<\arg \left[\prod_{s=1}^{n_{j}}\left(\frac{z_{1}-u_{1}}{z_{1}-a_{j s}}\right)\right]<n_{j}\left(\pi-\mu_{j}\right),
\end{array}
$$

for every $j=1,2, \ldots, p$. Using these inequalities and the value of $\mu_{j}$ from (2.5), we conclude that

$$
\gamma<\arg \left[\prod_{t=1}^{n_{j}-1}\left(\frac{z_{1}-b_{j t}}{z_{1}-u_{1}}\right) \cdot \prod_{s=1}^{n_{j}}\left(\frac{z_{1}-u_{1}}{z_{1}-a_{j s}}\right)\right]<\pi-\gamma
$$

In view of this and the hypotheses on $\alpha_{j}$, we obtain

$$
0<\arg \left[\frac{\alpha_{j}}{2} \cdot \prod_{t=1}^{n_{j}-1}\left(\frac{z_{1}-b_{j t}}{z_{1}-u_{1}}\right) \cdot \prod_{s=1}^{n_{j}}\left(\frac{z_{1}-u_{1}}{z_{1}-a_{j s}}\right)\right]<\pi
$$


for every $j=1,2, \ldots, p$. Finally, inequalities (2.8) and (2.9) imply that the imaginary parts of each term on the left-hand side of (2.7) are positive. This contradicts the fact that $z_{1}$ satisfies (2.7). Therefore, every zero $z_{k}$ of $S(z)$ lies in the region $K$. This completes the proof.

THEOREM (2.2). Under the hypotheses and notations of Theorem (2.1), the zeros of each Van Vleck polynomial V(z), associated with the differential equation (1.2), lie in the region $K$.

Proof. Let $t_{k}$ be a zero of a Van Vleck polynomial $V(z)$ corresponding to an $n$ th-degree Stieltjes polynomial $S(z)$, associated with (1.2). Using abbreviations (2.1) and (2.2), we know [10, Lemma (2.2)] that every zero $t_{k}$ of $V(z)$, if not an $a_{j s}$, is either a zero of $S^{\prime}(z)$ or satisfies the equation

$$
F\left(t_{k}\right)+\sum_{j=1}^{n-1} \frac{1}{t_{k}-z_{j}^{\prime}}=0
$$

$z_{j}^{\prime}(1 \leqslant j \leqslant n-1)$ being the zeros of $S^{\prime}(z)$.

If a zero $t_{k}$ of $V(z)$ is an $a_{j s}\left(1 \leqslant j \leqslant p, 1 \leqslant s \leqslant n_{j}\right)$, then $t_{k}$ is in $K$ and we are done. If a zero $t_{k}$ of $V(z)$ is a zero of $S^{\prime}(z)$, then Theorem (2.1) and Lucas' theorem [5, Theorem $(6,2)],[4]$ imply that $t_{k}$ is in $K$, and the theorem follows.

In order to prove the theorem for the case when $t_{k} \neq a_{j s}(1 \leqslant j \leqslant p, 1 \leqslant s$ $\left.\leqslant n_{j}\right)$ and $S^{\prime}\left(t_{k}\right) \neq 0$, we suppose (on the contrary) that some zeros of $V(z)$ lie outside $K$. Arguing as in the proof of Theorem (2.1), we can find a zero (say, $t_{1}$ ) of $V(z)$ outside $K$ and a member $E_{e}$ (with eccentricity $e$ ) of $\mathscr{F}$ passing through $t_{1}$ such that all zeros of $V(z)$ lie on or inside $E_{e}$. Our previous diagram (Figure 1) and construction remains the same except that $t_{1}$ replaces $z_{1}$. (2.10), for $k=1$, can be written as

$$
\sum_{j=1}^{p} \alpha_{j}\left\{\prod_{t=1}^{n_{j}-1}\left(\frac{t_{1}-b_{j t}}{t_{1}-u_{1}}\right) \cdot \prod_{s=1}^{n_{j}}\left(\frac{t_{1}-u_{1}}{t_{1}-a_{j s}}\right)\right\}+\sum_{j=1}^{n-1} \frac{t_{1}-u_{1}}{t_{1}-z_{j}^{\prime}}=0
$$

where $u_{1}$ is a point on the tangent to the ellipse $E_{e}$ at the point $t_{1}$. Since the points $z_{j}^{\prime}$ lie on or inside $E_{e}$ (but not on $T$ ), we obtain

$$
0<\arg \left(\frac{t_{1}-u_{1}}{t_{1}-z_{j}^{\prime}}\right)<\pi \quad(j=1,2, \ldots, n-1) .
$$

Replacing $z_{1}$ by $t_{1}$ in inequality (2.9), established in the proof of Theorem (2.1), we obtain also the inequality

$$
0<\arg \left[\alpha_{j} \cdot \prod_{t=1}^{n_{j}-1}\left(\frac{t_{1}-b_{j t}}{t_{1}-u_{1}}\right) \cdot \prod_{s=1}^{n_{j}}\left(\frac{t_{1}-u_{1}}{t_{1}-a_{j s}}\right)\right]<\pi
$$

for every $j=1,2, \ldots, p$. Now inequalities (2.12) and (2.13) contradict (2.11). Hence, every zero $t_{k}$ of $V(z)$ in this case also lies in the region $K$. This completes the proof of our theorem. 
An immediate consequence of the above theorems is the following result due to Marden [6, Theorem 6(b)] concerning (1.1).

COROLlary (2.3). If $\left|\arg \alpha_{j}\right| \leqslant \gamma<\pi / 2$ and if all the points $a_{j}$ lie on the line segment joining the points $c_{1}$ and $c_{2}$, then the zeros of every Stieltjes polynomial and the zeros of every Van Vleck polynomial, associated with the differential equation (1.1), lie in the region $K_{1}$ given by

$$
K_{1}=\left\{z|| z-c_{1}|+| z-c_{2}|\leqslant| c_{1}-c_{2} \mid \cdot \sec \gamma\right\} .
$$

Proof. If we put $n_{j}=1$ for every $j$ (so that $q=1$ ), then (1.2) reduces to (1.1), with the constants $a_{j 1}$ (in (1.2)) corresponding to the constants $a_{j}$ occurring in (1.1). Under this reduction, the region $K$ of the previous theorems is indeed the region $K_{1}$ and Corollary (2.3) is fairly obvious.

The above corollary expresses, for $\gamma=0$, the results stated in Theorems 1(a) and 1(b) in Marden [6], due, respectively, to Stieltjes [8] and Van Vleck [9].

The author is thankful to the referee for pointing out the fact that Corollary (2.3) is a result due to Marden.

\section{REFERENCES}

1. M. Bôcher, Über die Reihenentwickelungen der Potentialtheorie, Göttingen, 1894, pp. 215-218.

2. E. Heine, Handbuch der Kugelfunctionen, Bd. I, 2nd ed., Springer, Berlin, 1878, pp. 472-476.

3. F. Klein, Über lineare Differentialgleichungen der zweiten Ordnung, Göttingen, 1894, pp. 211-218.

4. F. Lucas, Propriétés géométriques des fractions rationnelles, C. R. Acad. Sci. Paris 77 (1874), 431-433; ibid 78 (1874), 140-144; ibid. 78 (1874), 180-183; ibid. 78 (1874), 271-274.

5. M. Marden, Geometry of polynomials, 2 nd ed., Math. Surveys, no. 3, Amer. Math. Soc., Providence, R.I., 1966. MR 37 \# 1562.

6. - On Stieltjes polynomials, Trans. Amer. Math. Soc. 33 (1931), 934-944.

7. G. Pólya, Sur un théorème de Stieltjes, C. R. Acad. Sci. Paris 155 (1912), 767-769.

8. T. J. Stieltjes, Sur certains polynômes qui verifient une équation différentielle, Acta Math. 6-7 (1885), 321-326.

9. E. B. Van Vleck, On the polynomials of Stieltjes, Bull. Amer. Math. Soc. 4 (1898), 426-438.

10. N. Zaheer and M. Alam, On the zeros of Stieltjes and Van Vleck polynomials, Trans. Amer. Math. Soc. (to appear).

Department of Mathematics, Aligarh Muslim University, Aligarh-202001, India. 